\title{
Size matters: exploring the links between food portion sizes and diet quality in Irish children
}

\author{
Jacqueline Lyons
}

School of Food and Nutritional Sciences, UCC

\section{Bigger portions: what's the big deal?}

Food portions have increased over the last 20 years. This has been shown to be true for the foods we eat at home, those consumed at restaurants and fast-food outlets, and for foods sold in packages. Along with this increase in food portion sizes has been an increase in both child and adult obesity, leading to the obvious question: could larger food portion sizes be contributing to the obesity epidemic?

The World Health Organisation (WHO) has cited large portion sizes as a 'possible' dietary risk factor for obesity, which according to their definition means that 'more trials are needed to support the tentative associations'. Their caution is based on the fact that the evidence comes mainly from cross-sectional studies, which typically include a large number of individuals but are essentially observational, and therefore cannot conclude that the relationship between large portion sizes and obesity is a causative one. The WHO acknowledges that evidence based on clinical and laboratory investigations has been supportive, but realistically the controlled feeding studies that have shown that larger portion sizes increase one's energy intake at that meal cannot ethically be continued to show that larger portion sizes may cause weight gain to the point of obesity. So, the causative relationship between portion sizes and obesity, although inherently assumed true by many and a much-loved media topic, is something of a closed avenue. Indeed, it may be more relevant to investigate how food portions of different sizes impact on other aspects of one's diet, and where better to examine this than among children, who are not just our future but the future users of our stretched health services.

\section{Previous research}

Studies have shown that young children, unlike the rest of the population, tend not to massively overeat. During the early years of life, eating tends to be driven by hunger and satiety cues alone, and, unlike teenagers and adults, young children have been shown to balance their energy intakes over a 24 hour period, such that high energy intake at one meal is typically followed by low energy intake at the next, and vice versa. As children 
grow, however, they begin to eat in response to external cues such as the presence of palatable food, the time of day and the social situation, and so, inevitably, food portion sizes begin to influence energy intake in children, typically from about 4 years old. Aside from influencing energy intakes, however, little attention has been paid to how food portion sizes may influence other aspects of dietary quality in children, such as intakes of fat, iron, fibre and other important nutrients. This research 'gap' has provided an opportunity for the current work to be carried out.

\section{The Irish link}

The aim of the current work was to use data from the Irish National Children's Food Survey (NCFS) to investigate how different portion sizes of a range of foods impact upon other markers of dietary quality on the days that they are consumed. The NCFS was carried out between 2003 and 2004 to establish a database of habitual food and drink consumption in a representative sample of Irish children aged 5 to 12 years. Five hundred and ninetyfour children from across the country kept a detailed record of all the foods, drinks and supplements they consumed over a seven-day period, with help from their parents. In addition to this, anthropometric data (i.e., height, weight, leg length, waist and hip circumference), physical activity data and health and lifestyle characteristics were collected from both the child and their parents. Seventy-six per cent of all food items consumed on the survey were weighed using a digital scales, with a further $11 \%$ assigned weights from manufacturer's information (e.g., one can of Coke $=330 \mathrm{ml}$ ). As dietary surveys go, this represents a large proportion of reliable weights, making the data particularly suitable for an analysis involving portion sizes.

For the current work, portion size ( $\mathrm{g}$ ) was defined as the weight of food consumed per eating occasion, and was estimated for each day that the food was consumed. Where a food was consumed on more than one occasion on one day, the largest portion size was assigned. The portion size data were then split into 'thirds' (small, medium and large portions), with aspects of dietary quality compared across the three groups.

Because the same portion of breakfast cereal may be considered a 'large' portion for a 5 -year-old girl but a 'small' portion for a 12-year-old boy, simply splitting the portion size data into 'small', 'medium' and 'large' segments could not accurately reflect how different portion sizes affect other aspects of the diet. To get around this, each food type included for analysis was individually examined to see whether there were significant differences in the portion weights consumed by boys and girls, or by younger and older children. (Unsurprisingly, it was seen that boys typically ate larger portions than girls of the same age, and that portion weights of most foods increased with age.) Wherever significant differences were noted, the data were stratified (arranged in layers), meaning that the resulting three portion size groups represented relatively 'small', 'medium' and 'large' portions for that particular food. In other words, those in the 'small' portion group included a 
mix of boys and girls of different ages, all of whom consumed 'small' portions of the food relative to their peers of the same age group and gender. Once the portion weights were arranged into these three groups, it was possible to look at mean intakes of a selection of nutrients on the days in which the foods were consumed, to see whether differences occurred between the groups.

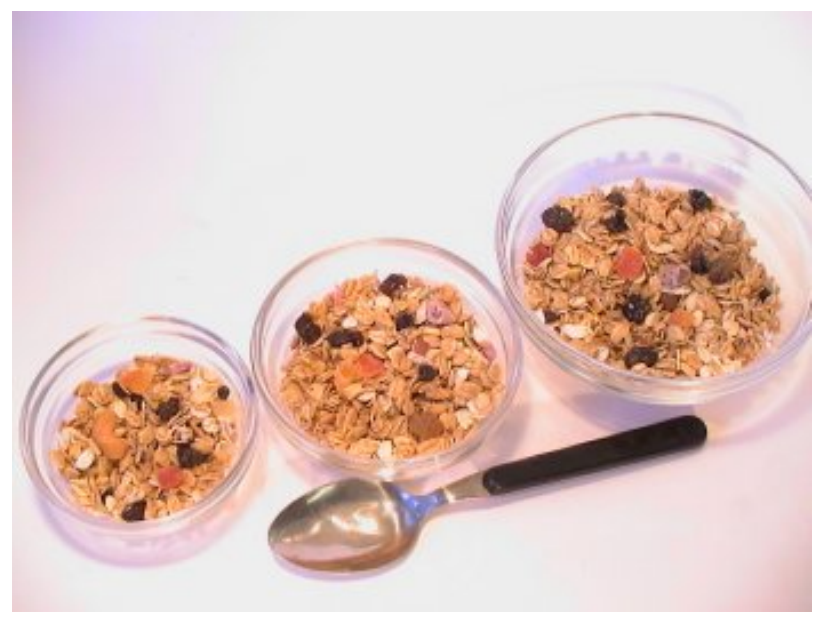

Figure 1: Food portions were split into three groups for analysis

(Photo with permission from www.calorieking.com.au)

\section{Key findings}

The analysis showed clear evidence for the 'sugar-fat seesaw' phenomenon, in that children who ate larger portions of high-sugar foods (e.g., sugary sweets and sugar-sweetened beverages) were seen to have decreased intakes of fat on that day, and those who ate larger portions of high-fat foods (e.g., pizza and chips) had decreased sugar intakes on that day. This finding has significant implications for interventions aimed at improving the quality of Irish children's diets. It suggests that, as recommended by experts in this area, a key measure in enabling children to meet their fat and saturated fat goals would be to encourage the substitution of high-fat foods with starchy foods like potatoes, rice or pasta; starch, essentially, being a healthier and less refined form of sugar.

Table 1 provides a snapshot of my findings. It describes the percentage of total energy provided by saturated fat (mean values) on the days in which the named foods were consumed. It is recommended that children obtain no more than $11 \%$ of their total daily energy from saturated fat, with increasing intakes known to increase the risk of coronary heart disease. We can see from the table that all of the mean values exceed this, and so in general, this population goal is not being achieved by Irish children. As we look from 'small' to 'medium' to 'large' on the table, it is possible to see how the percentage of energy from saturated fat changes as the portion size of each of the foods examined increases. The column labelled 'P' uses arrows to denote statistically significant increases 
Table 1: Saturated fat as a percentage of total energy on the days in which the foods were consumed

\begin{tabular}{|l|c|c|c|c|}
\hline & \multicolumn{4}{|l|}{ Portion size groupings } \\
\hline Foods & Small & Medium & Large & $\mathrm{P}$ \\
\hline Pizza & 13.5 & 14.4 & 14.4 & \\
\hline White bread and rolls & 14.4 & 14.0 & 13.3 & $\downarrow$ \\
\hline Brown bread and rolls & 14.4 & 13.7 & 13.3 & $\downarrow$ \\
\hline Boiled potatoes & 14.7 & 14.1 & 13.2 & $\downarrow$ \\
\hline Chips & 14.0 & 13.9 & 13.4 & \\
\hline Breakfast cereals & 14.2 & 14.0 & 13.5 & $\downarrow$ \\
\hline Fruit & 14.4 & 13.7 & 13.2 & $\downarrow$ \\
\hline Vegetables & 14.5 & 13.8 & 13.8 & $\downarrow$ \\
\hline Roast meats & 14.1 & 13.8 & 13.6 & \\
\hline Frying meats & 14.7 & 14.8 & 15.0 & \\
\hline Eggs, cooked & 15.0 & 14.2 & 15.9 & $\uparrow$ \\
\hline Milk & 13.1 & 14.4 & 15.2 & $\uparrow$ \\
\hline Cheese & 14.5 & 15.1 & 16.7 & $\uparrow$ \\
\hline Chocolate & 14.2 & 14.8 & 15.7 & $\uparrow$ \\
\hline Sugary sweets & 14.2 & 13.6 & 12.6 & $\downarrow$ \\
\hline Fruit juice & 13.8 & 13.8 & 13.4 & \\
\hline
\end{tabular}

or decreases in saturated fat as a percentage of total energy as portion size increases. The table, therefore, shows that larger portions of white and brown bread, boiled potatoes, breakfast cereals, fruit, vegetables and sugary sweets are associated with a reduction in saturated fat on the days in which they are consumed. An increase in saturated fat is observed with increasing portions of eggs, milk, cheese and chocolate.

\section{Discussion}

The issue of food portion size is becoming ever more topical. This has not gone unnoticed by food manufacturers, who have come up with various 'helpful' techniques to discourage consumers from overeating: $150 \mathrm{~g}$ crisp packets are now marketed as 'sharing' packs, the idea presumably being to stop eating when you reach $35 \mathrm{~g}$, which is the standard pack size. Multipack crisps offer another opportunity to consume a smaller portion (typically $25 \mathrm{~g}$ versus $35 \mathrm{~g}$ ); however, to access smaller pack sizes it is necessary to buy a stock of them together, and willpower is then required to ignore the remaining goodies. Fast-food outlets are aware of the portion size issue too, and while it is still entirely possible to purchase enormous meals, there does seem to be some acknowledgement of the issue, as witnessed by McDonald's recent withdrawal of its supersize menu option.

While the issue of food portion size seems to be growing in importance, it tends to dwell 


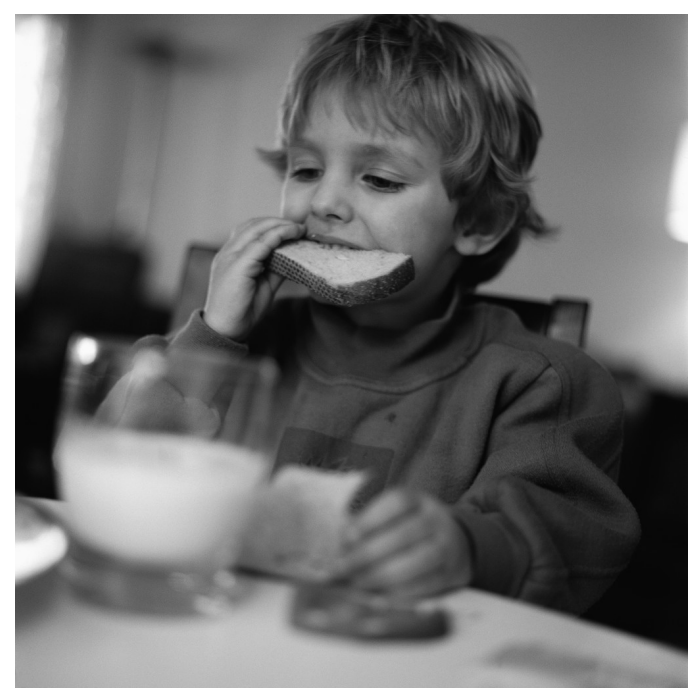

Figure 2: Bread and milk — still staples among Irish children but portion size matters

on the portion sizes of less healthy foods, giving less attention to the portion sizes of healthier foods and the effects that these may have on the overall diet. The current work takes a somewhat new angle in investigating associations between portion sizes of a wide variety of foods, both 'healthy' and 'less healthy', that are commonly consumed by Irish children and how these may impact on the diet.

\section{Conclusion}

In May 2011, the Irish Medical Times reported the plans of the HSE to tackle the so-called 'Big Six' diseases, that is, the six main chronic diseases suffered in Ireland which account for up to $90 \%$ of chronic disease morbidity, with associated lost work days and long-term healthcare expenses. The diseases are stroke, acute coronary syndrome, heart failure, chronic obstructive pulmonary disease (COPD), asthma and diabetes. Dietary factors play a major role in the onset of many of these illnesses, or, put more positively, it is possible to prevent the occurrence of a significant proportion of these illnesses by focusing on improved nutrition and maintenance of a healthy weight, particularly from childhood.

Findings such as mine are only relevant if they can be applied to real life. Food portions have been cited internationally as a 'crucial issue' to be included in food based dietary guidelines; however, the most recent dietary guidelines for Irish children are somewhat vague in recommending that 'a balanced intake of all foods in the appropriate amounts should ensure the optimal development of a child'. Because my findings describe specific associations between food portions and other important markers of dietary quality, they should be useful in the development of more practical food-based dietary guidelines aimed at Irish children in the future, for which there is clearly a need. supervision of Professor Albert Flynn. The author wishes to acknowledge funding from the 
Size matters: exploring the links between food portion sizes and diet quality in Irish children

JACQUELINE LYONS

Irish Government under the National Development Plan 2000-2006. 